\title{
Discovery of a Novel Sarcocystis Infection in an Immunocompromised Patient
}

\author{
Cynthia S. Goldsmith ${ }^{1}$, Maureen G. Metcalfe ${ }^{1}$, Norman J. Pieniazek ${ }^{2}$, Alexandre Dasilva ${ }^{2}$, Wun-Ju \\ Shieh $^{1}$, and Sherif R. Zaki ${ }^{1}$. \\ ${ }^{1}$ Infectious Diseases Pathology Branch, National Center for Emerging and Zoonotic Infectious \\ Diseases and ${ }^{2}$ Parasitic Diseases Branch, Division of Parasitic Diseases and Malaria, Center for \\ Global Health, Centers for Disease Control and Prevention (CDC), Atlanta, GA 30333
}

Sarcocystis is a protozoan parasite that can infect numerous species of animals, including humans. Most cases have been found in persons living in tropical or subtropical environments. In human muscular infections, the source of the protozoa can be water or food contaminated with feces from an infected animal. Symptoms can take weeks to months to develop, and might last months to years; however, many patients are asymptomatic.

In 2010, a 32-year old woman who had travelled to Colorado after living in Kenya and Sudan became ill with acute leukemia. She was treated with chemotherapy and suffered from congestive heart failure after each treatment. She then received a bone marrow transplant but died of congestive heart failure approximately two months later. Cardiac tissue showed numerous collections of micro-organisms, and toxoplasmosis and Leishmania were in the differential diagnosis. Subsequently, tissues were sent to CDC for definitive diagnosis.

Histopathologic examination of the heart and lung revealed small round protozoal organisms in cystic structures, located in vessels, perivascular areas, and myocytes. Electron microscopy showed organisms without kinetoplasts, a feature that is seen in Leishmania protozoa which characteristically infect macrophages. Rather, round to oval protozoa were found in large cysts within endothelial cells lying between the cardiac muscle fibers (Figs. 1, 2, 3). These merozoites contained a nucleus, a conoid with accompanying microtubules, micronemes, rough endoplasmic reticulum, a Golgi complex, mitochondria, and lipid. Conoids are characteristic of the phylum Apicomplexa, which includes Toxoplasma gondii and Cryptosporidium parvum. The protozoa replicated by endopolygeny, a form of asexual reproduction (Fig. 4). DNA was extracted from formalin-fixed, paraffin-embedded heart tissue and PCR with generic 18rRNA primers was performed. Amplicons produced were sequenced and preliminary DNA sequencing analysis revealed evidence of a novel Sarcocystis species.

The patient was most likely asymptomatic from her infection with Sarcocystis which she likely contracted in eastern Africa. Immunosuppression presumably played a role in the development of the protozoa and a systemic infection. Histopathologic and electron microscopic examination, coupled with PCR analysis, lead to the discovery of this novel Sarcocystis. 


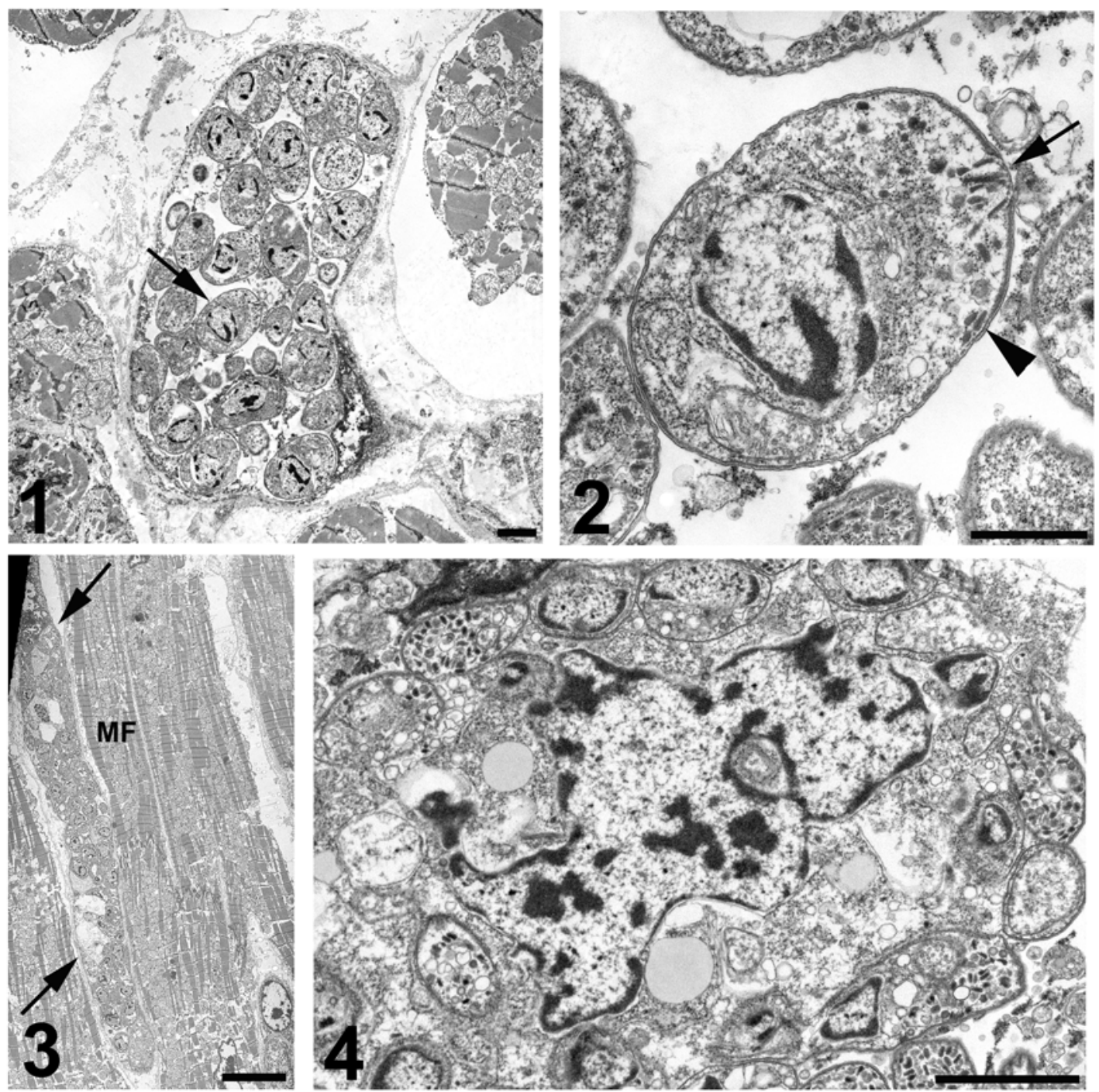

Figure 1. A cyst in cardiac tissue, filled with round to oval protozoa. Bar, 2 um. Figure 2. Higher magnification of the protozoa pointed to in figure 1, showing the characteristic conoid structure (arrow) and micronemes (arrowhead). Bar, $1 \mathrm{um}$. Figure 3. A large cyst (between arrows), approximately $68 \mathrm{um}$ in length, is seen between the muscle fibers of the heart (MF). Bar, $10 \mathrm{um}$. Figure 4. Large, irregular nucleus in a cyst, indicative of replication by endopolygeny. Bar, 2 um.

Disclaimer: The findings and conclusions in this report are those of the authors and do not necessarily represent the official position of the Centers for Disease Control and Prevention. 